\title{
Cochlear implants in children
}

\author{
Devanand Jha ${ }^{1}$ \\ Sri Lanka Journal of Child Health, 2005; 34: 75-8
}

(Key words: cochlear implants, children)

\section{Introduction}

A cochlear implant is a small complex electronic device that can help to provide a sense of sound to a person who is profoundly deaf or severely hard of hearing. An implant does not restore or create normal hearing. Instead, under appropriate conditions, it can give a deaf person a useful auditory understanding of the environment and help the recipient to understand speech. Cochlear implants are now firmly established in the management of the profoundly hearing impaired children. Ever since the PDA approved the Nucleus 22 channel cochlear implant in 1990 for children between 2-17 years of age, thousands of children have received the cochlear implant. According to the PDA 2002 data, approximately 59,000 people worldwide have received implants, among them 13.000 adults and 10,000 children from the USA. The key to successful implant programme lies in careful patient selection, skilful surgery and experienced rehabilitation.

All cochlear implants have two components. The internal component is implanted and the external component is worn outside. The external component consists of a microphone, an external transmitter and a signal processor or speech processor. The microphone picks up sound from the environment. The speech processor selects and arranges sounds picked up by the microphone. The internal component consists of electrodes that are implanted into the cochlea, and a receiver that is embedded in the temporal bone behind the ear. The receiver stimulator receives signals from the speech processor and converts them into electrical impulses. The electrodes collect the impulses from the stimulator and send them to the brain. The incoming sound is analyzed by the signal processor and computed into fundamental acoustical information that represents key elements of human speech. The analysis results in coding of the appropriate electrode, current amplitude and stimulus rate. The processed signal is

${ }_{\text {Consultant ENT Surgeon, Apollo Hospitals, }}$,
Colombo

sent transdermally via radio frequency transmission to the internal receiver. The message is decoded such that separate bipolar pairs of electrodes are activated to stimulate segments of the auditory nerve ${ }^{1}$.

Candidate selection: The selection criteria for. Cochlear implants in children are as follows ${ }^{2}$ :

- Twelve months of age or older (PDA recommendation 2002). However, in specific circumstances, even before one year of age the child can be implanted as when meningitis is the cause of hearing loss. Meningitis causes ossification in cochlea as time passes rendering electrode insertion more difficult.

- Profound bilateral sensorineural hearing loss.

- No appreciable benefit from hearing aids.

- No medical contraindications.

- High motivation and appropriate expectations.

- Enrollment in a programme that emphasizes development of auditory skills.

\section{Preoperative evaluation:}

Once a child is referred for cochlear implantation, a multidisciplinary evaluation should begin The cochlear implant team should include a surgeon, an audiologist, a speech-language pathologist and teacher for the deaf. The preimplant begins with an audiologic evaluation. Unaided and aided hearing thresholds arc obtained with the use of conventional amplification. Potential implant candidates must have bilateral, profound sensorineural hearing loss with a pure tone and unaided threshold equal to or greater than $95 \mathrm{~dB}$. In children less than 6 years of age, auditory brainstem testing is performed frequently to confirm the hearing loss. Every effort is made to establish the aetiology of deafness. With few exceptions, experience with cochlear implant demonstrates that auditory neural elements that can 
be stimulated seem to be present, regardless of whether the cause of deafness is congenital or acquired $^{3}$.

Prior to implantation, particular attention is paid to the tympanic membrane and the middle ear. An otologically stable condition should be present prior to implantation. If the child has recurrent otitis media, the condition should be treated first. Grommets can be inserted but they should be removed 4-6 weeks prior to implantation.

\section{Radiologic assessment}

High resolution thin section CT scanning of the cochlea and temporal bone is performed to assess the feasibility of implanting. It assists the decision on which ear to implant and ensures that the inner ear morphology is normal. Congenital cochlear abnormalities do not necessarily preclude implantation, but need to be identified pre operatively in order to assist in surgical planning. Absolute contraindications to implantation include cochlear aplasia or absence of the auditory nerve ${ }^{4}$. When the internal auditory canal is less than $2 \mathrm{~mm}$ in size, magnetic resonance imaging should be performed to confirm the presence of the cochlear nerve.

\section{Psychologic and social considerations}

The parents and family members should become an integral part of the implant team. The parents need to understand that a tremendous amount of post operative rehabilitation is carried out at home. At the current time, psychologic and social considerations for a successful candidate include ${ }^{5}$ :

- No evidence of severe organic brain damage.

- No evidence of psychosis.

- No evidence of mental retardation.

- No behavioural or personality traits that would make completion of the programme unlikely.

- No unrealistic expectations about the goals of the implant.

- A pattern of parent/child interaction which indicates that the family will be able to follow the post implant rehabilitative programme effectively.

\section{Surgical procedure}

In children flap design is crucial because of delicate tissues and small dimensions. A post auricular incision is made and flaps elevated. A palvas flap is elevated based anteriorly. Cortical mastoidectomy is done and posterior tympanotomy is done to open into the middle ear and expose the round window niche. The bed of the receiver is drilled out posterior to the cortical mastoidectomy and tie down holes are drilled to keep the implant in place. A cochleostomy is done through the posterior tympanotomy window and the electrodes are inserted in the cochlea. The receiver is anchored with prolene and the wound closed in layers. Once the wound has healed and flap edema has resolved, approximately 4-6 weeks later, fitting and mapping of the signal processor begins.

\section{Rehabilitation}

Approximately 4-6 weeks after surgery the child is seen for the initial mapping and tune-up session. During this session the child is fitted with the: external equipment and the parents and child are given instructions in the maintenance of the unit. Threshold and comfort levels are obtained for each of the active electrodes. The threshold is defined as the minimum amount of current that is required for the child to indicate that the sound is perceived ${ }^{6}$.

Every child is unique with respect to mapping. The parents' active participation during the sessions helps them to realize the benefits and limitations of the implant and gives them practice in working with the child. Depending on the device and the child's age and needs, the rehabilitation process may require daily sessions for 1-2 weeks until the map is set. The time commitment on part of the parents must be understood before the child undergoes implantation.

\section{Results}

Several factors including age at the time of deafness, age at the time of implantation, duration of deafness, status of the remaining nerve fibres, educational setting, type of implant, and the length of time with the implant, all play a role in the success of the implant.

Children with congenital deafness and children with prelingually acquired meningitic deafness achieve similar auditory performance as long as the cochlear nerve is intact ${ }^{7}$. Children with a memory of previous auditory experience and a short period of deafness have a distinct advantage over children who are prelingually hearing impaired. Postlingual deaf subjects showed dramatic improvements on all 
perceptual tests after only 6 months of implant use because of advantage of previous hearing ${ }^{8}$. Prelingual hearing impaired children show large improvement in speech perception skills, but over a longer, protracted course. Perceptual performance increases on average with each succeeding year after implantation. The most important factor contributing to individual differences among implant users is the amount of experience that users have with the device ${ }^{9}$.

In children with prelingual hearing loss who received implants, Gantz et al reported that 4 years following implantation $80 \%$ of children achieved open-set, sound-only, word understanding. Speech perception skills continue to develop and do not reach a plateau ${ }^{8}$.

Age at the time of implantation is important. It is likely that the auditory and the central nervous system have a critical period of learning, and these systems must be stimulated before a certain age in order to achieve speech perception ${ }^{8}$. The age at which auditory information provided by cochlear implants becomes less useful to deaf individuals is unknown. Speech production results in a limited number of children with prelingual hearing loss, suggesting that children with early onset deafness (prior to 4 years of age) demonstrate more improvement of speech production skills than those who receive an implant after 10 years of age ${ }^{10}$. However, results have shown that prelingually deaf children up to the age of 13 years of age can obtain substantial speech perception from multichannel implants ${ }^{8}$. Because older children, on the whole, tend to perform more poorly, they should be assessed in a much more intensive manner to ensure proper selection of the appropriate candidate.

\section{Living with an implant}

There are a few things that children with implants should not do. They should avoid contact sports such as boxing where they might be hit on the head. Swimming is rarely a problem because the external part of the implant can be taken off while swimming.

\section{Experience in Sri Lanka}

Six patients have undergone cochlear implant surgery in Sri Lanka in the age range of 1 year to 56 years. Two of them are postlingual and the other four are prelingual patients. They are undergoing harbilitation and rehabilitation and are doing well.

\section{Conclusions}

Currently children as young as 10 monih of age with profound deafness are candidates for implantation. Auditory performance with cochlear implant varies among individuals, but data indicate that performance is better in children who have shorter duration of deafness, who have acquired speech and language before the hearing loss, and who have received the implant before the age of 6 (if prelingual). Auditory performance does not seem to be affected by the aetiology of the hearing loss. Access to optimal education and. rehabilitation services is vitally important for children to maximize the benefits available from cochlear implantation. Cochlear implants continue to improve and current generation of intracochlear, muitichannel implants with spectrally based speech processors provide a substantial improvement over devices of the previous generation. Advances that have been made in improving speech perception in cochlear implant users should continue to improve with changes in electrode design. and signal processing strategies. In future it may be possible to implant the whole external and internal part behind the ear under the skin.

\section{References}

1. Kveton J, Balkany T. American academy of otolaryngology- head and neck surgery subcommittee on cochlear implants: Status of cochiear implantation in children. $J$ Pediatr 1991; 118: 1-7.

2. Miyamoto $\mathrm{R}$ T. Cochlear Implants in children. In: Bluestone C, Stool S, Kenna M, editors. Pediatric Otolaryngoglogy. 3rd ed. Philadelphia: Saunders, 1996; 671-5.

3. Hinojosa R, Marion M. Histopathology of profound sensorineural deafness. Ann NY Acad Sci 1983; 405: 459-84.

4. Shelton C, Luxford W, Tonokawa L, Lo W, House W. The narrow internal auditory canal in children: A contraindication to cochlear implants. Arch Otolaryngol Head Neck Surg 1989; 100:227-31.

5. Hartrampf R, Lesinski A, Allum D, Dahm M, Lenarz T. Reasons for rejected candidacy for cochlear implantation in children. $A d v$ Otolaryngol 1995; 50:14-8. 
6. Pansier S, Chute P. Nevins M. Pediatric cochlear implants: Surgical and rehabilitative issues. Highlights of instructional Courses, num 7. St.Louis: Mosby Yearbook, 1994; 145-54.

7. NIH Consensus Development Panel on Cochlear Implants in Adults and Children. Cochlear implants in adults and children. JAMA 1995; 274: 1955-60.

8. Grantz B, Tyler R, Woodworth G, Tye Murray N, Fryauf-Bertschy H. Results of multichannel cochlear implants in congenital $\&$ acquired pre-lingual deafness in children. 5 year follow-up Am J Otol 1994; 15:1-7.

9. Miyamoto R T, Kirk K, Robbins A. Todd S, Riley A. Speech perception and speech production skills of children with multichannel cochlear implants. Acta Otolaryngol 1996; 116. 240-3.

10. Staller S, Beiter A, Brimacombe J. Mecklenburg D, Amdt P. Pediatric performance with the Nucleus 22 channel cochlear implant system. Am J Otol 1991; 12: 126-36.

\section{Editorial Comments}

Cochlear implantaton services are now available in Sri Lanka.

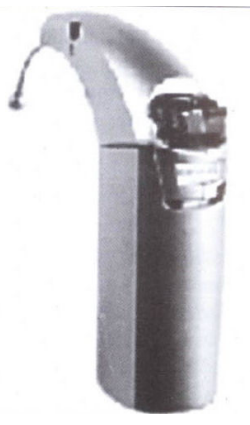

Nucleus contour device (implantable part)

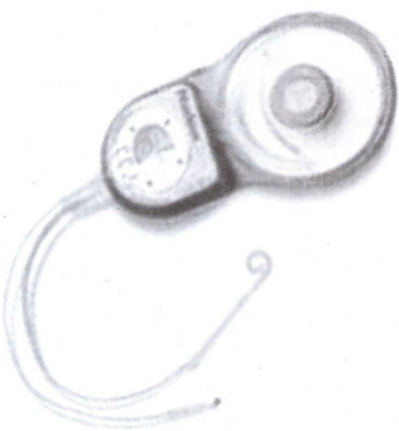

Nucleus Espirit 3G Speech processor 